\section{Stilblüten des FCKW-Verbotes}

\author{
Bürokratische Zwänge sind in Deutschland besonders verbreitet. \\ Diese Tatsache gereicht bei ausländischen Gästen, die man in \\ die Tiefen deutschen Verwaltungsrechts einführt, oftmals zu \\ schmunzelnden Kommentaren bis zu ausgeprägten Lachsalven. \\ Beim jüngsten Fall medizinbürokratischen Verwaltungswahns \\ bleibt jedoch vielen betroffenen Patienten und Allergologen das \\ Lachen im Halse stecken.
}

A usgangspunkt ist die „FCKW-Halon-Verbots-Verordnung", deren $\$ 2$ das „Inverkehrbringen von FCKWhaltigen Druckgaspackungen" verbietet. Im speziellen Falle wurde von einer angesehenen deutschen Universitätsklinik das Präparat Primatene ${ }^{\circledR}$ Mist einem hochgradig betroffenen Insektengiftallergiker für die Notfallapotheke verordnet mit dem Hinweis auf der Rezeptur, dass ein vergleichbares Präparat in Deutschland nicht zur Verfügung stehe. Diese Tatsache ist zweifelsfrei auch für Bürokraten nicht zu leugnen und hat in Allergologenkreisen bereits seit geraumer Zeit für Bedenken hinsichtlich einer adäquaten Notfalltherapie von anaphylaxiegefährdeten Patienten - vor allem Hymenopterengiftallergiker - geführt.

Dieses Rezept löste eine bürokratische Kettenreaktion mit enormen Unkosten aus, die für die zum sparsamen Umgang mit Ressourcen angehaltene Ärzteschaft ärgerlicher nicht sein kann. Die Angelegenheit gipfelte in einem Schreiben der Bezirksregierung Braunschweig, aus dem nachfolgend einige wichtige Passagen zitiert werden:

„Nach $\mathbb{} 2$ FCKW-Halon-VerbotsVerordnung ist das Inverkehrbringen von FCKW-haltigen Druckgaspackungen (z. B. Primatene mist) verboten. Eine Zuwiderhandlung stellt nach $\mathbb{S} 9$ Abs. 1 FCKW-Halon-Verbots-Verordnung einen Straftatbestand dar. Das Verkehrsverbot von FCKW-haltigen Druckgaspackungen hat gegenüber der Regelung des $\mathbb{I} 73$ Abs. 3 Arzneimittelgesetz $(A M G)$, die den Einzelimport nicht zugelassener Arzneimittel durch Apotheken erlaubt, Vorrang.

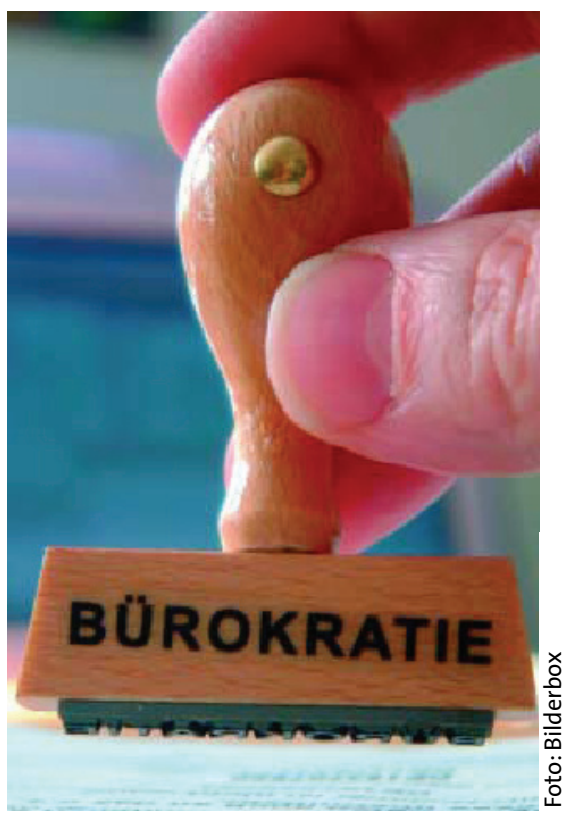

Deutsche Medizinbürokratie - Lachen oder Weinen?

Ausnahmen von diesem Verbot wurden nur dem Bundesinstitut für Arzneimittel und Medizinprodukte (BfArM) im Rahmen der Entscheidung über die Zulassung eines Arzneimittels zugestanden. Für die Erteilung einer verwaltungsbehördlichen Ausnahmegenehmigung im Einzelfall, die z. B. den Einzelimport von ,Primatene mist' erlaubt, ist daher kein Raum.

Aus diesem Grund dürfen Verschreibungen über, Primatene mist' nicht mehr durch Apotheken beliefert werden.

Selbst die Berufung auf einen rechtfertigenden Notstand gem. \$3 34 Strafgesetzbuch (StGB) ist bei einer Einfuhr im ärztlich begründeten Einzelfall bei prophylaktischer Verordnung unzulässig. Ein solcher Notstand rechtfertigt bei einer gegenwärtigen, nicht anders abwendbaren Gefahr rechtswidriges Verhalten, nicht jedoch bei einer latenten, ggf. künftig eintretenden Gefahr."

Deutet man diese Äußerungen richtig, so darf bei bereits eingetretener Anaphylaxie Primatene ${ }^{\circledR}$ Mist verordnet und eingeführt werden, nicht jedoch für die Notfallapotheke der Betroffenen. Dies führt natürlich jeglichen Sinn einer Notfallapotheke ad absurdum. Wird man zukünftig den Sicherheitgurt erst anlegen dürfen, wenn man bereits mit dem Bremsen bei einem Auffahrunfall begonnen hat? Erschreckend ist natürlich die Tatsache, dass den betroffenen Ärzten hier unverhohlen mit strafrechtlichen Maßnahmen gedroht wird, obwohl eine sachgerechte und leitlinienkonforme Behandlung eines hochgradig gefährdeten $\mathrm{Pa}$ tienten erfolgt.

$\mathrm{Ob}$ dieser Fall nun eher zum Lachen oder zum Weinen anregt, mag jeder Leser für sich entscheiden.

Für den betroffenen Patienten bleibt jedoch eine klar erkennbare Unterversorgung und die Verunsicherung, für den Notfall nicht ausreichend versorgt zu sein.

Prof. Dr. Ludger Klimek, Wiesbaden

In memoriam

Am 4. November 2002 ist Dr. med. Helmuth Hase aus Braunschweig verstorben. Dr. Hase war Ehrenmitglied des ÄDA seit 1988.

Impressum „Der ÄDA informiert"

Verantwortlich für den Inhalt: Prof. Dr. Ludger Klimek, Wiesbaden Redaktion: Markus Seidl

Urban \& Vogel Medien und Medizin Verlagsgesellschaft $\mathrm{mbH} \& \mathrm{Co}$. KG Neumarkter Straße 43 81637 München

ÄDA-Geschäftsstelle: Service Systems Carin Fresle, Ursula Raab E-Mail:aeda@sersys.de 